\title{
The design and implementation of the management system of educational administration experiment
}

\author{
Zhang Jie ${ }^{1}$, Bian Wenyu ${ }^{1}$ \\ 1.Department of mathematics and computer, Zhangjiajie College of JishouUniversity, \\ ZhangjiajieHunan, China
}

Keywords:management system;design;educational administration; work flow

\begin{abstract}
Universities pay more attention to the cultivation of students' practical ability, change the traditional experimental curriculum arrangement mode, reform the experimental teaching management mode, and change to an open scientific and standardized experimental teaching management has important practical significance. At present, with the development of network information technology, the basic conditions for establishing a complete science experiment teaching management are becoming more and more mature. In order to realize the remote management of experimental teaching, remote management of experimental teaching, experiment resources and information sharing, the arrangement of the students is not limited by time and space.
\end{abstract}

\section{Introduction}

In recent years, the rapid development of computer network information, information technology in various industries has gradually increased, the university is responsible for the national scientific research work, which has a large number of scientific research personnel, information technology is not slow; on the other hand, comprehensive quality and innovation ability of the focus of public attention, training with strong practical ability, improve the experimental teaching reform and management has become an important task to cultivate students' comprehensive quality education. Under the background, how to use the modern information technology, to strengthen and realize the scientific management of experimental teaching, to develop an experimental teaching management system, to achieve the standard management of experimental teaching, optimize the allocation of resources, improve the quality of education is particularly important and meaningful; on the other hand, the current situation of domestic and international education information management, design a set of experimental teaching management platform, which is applicable to the base of the experimental management platform.

\section{System design pattern}

The experimental teaching management system is in a multi-tier Web application system, through the J2EE technology and the workflow application component to the application logic is divided into several levels, thus designing a multi-layer system structure based on J2EE. Which is divided into layers, including the presentation layer, business logic layer, data persistence layer and DAO (Access Object Data) model. Each level of their duties, complement each other, when entered the business system, the corresponding business logic by corresponding to the component technology, component technology again calls between components is achieved by the container to fully shared resources. Each level is described below:

Presentation layer: model view control (Model-View-Controller, MVC) is the fundamental and important role in the application of Web.

Business logic layer: inversion of control (IOC) model is a core part of the business logic layer. The first is the dependency injection is separated, once an object to be its target system in proper time will give it injection corresponding object, so as to achieve by calling relationships between them and the callee. So inversion of control (IOC) also called dependency injection (dependency injection) mode.

Data persistence layer: Data (DAO) model and Mapping Object-Relation (ORM) is the 
fundamental problem of Access Object (DAO), which is the fundamental of data persistence layer, so the two modes are the basic of data persistence layer, so that the business logic can be freed from the basic operation.

\section{System design}

Information system using JavaEE technology has a clear structure, high reusability, easy to expand, data security and other characteristics, and the system needs to have high scalability, and can be compatible with other application systems and upgrade to simple and convenient. Multi tier distributed structure system has a good application prospect, and the stability and security of the advantages of JavaEE technology, J2EE architecture technology to a large extent to meet the requirements of information management in large enterprises, but also take into account the development of the system. Using JavaEE technology to build the system using the layered technology design, MVC three layer, and then abstract the customer layer, through the hierarchical design, can reduce the coupling between modules, each layer uses component software development and integration. To analyze the correlation between the laboratory teaching management system and the correlation between the data, we decided to use the JavaEE based three layer $\mathrm{B} / \mathrm{S}$ architecture to improve the system performance and security.

The system will use the current mainstream B/S structure, based on the lightweight JavaEE multi-tier framework.

The system is divided into the teacher management subsystem, the student management subsystem, the laboratory management subsystem.

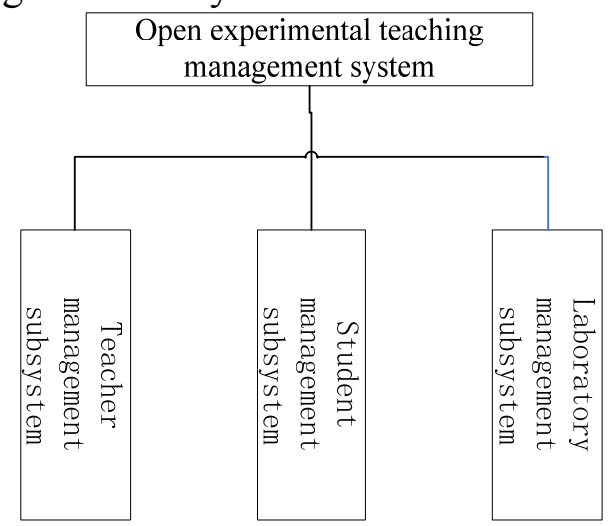

Figure 1 open experimental teaching management system function diagram

From the above needs analysis and data flow diagram can be drawn such a number of entities: teachers, students, courses, experimental projects, the experimental information, booking information, performance information, feedback information and other entities.

Teachers can manage courses and experimental projects, and the contents of the experimental project, teachers can publish announcement information, students can choose courses and experimental program to make an appointment, the students submit an appointment information, the teacher should be prepared for the experiment, students can also be a reference to the experimental arrangement, but also the students can manage the students' experimental results.

\section{System testing}

When the system is developed, the software life cycle, the overall system and the function of each sub module will be wrong, also will encounter many other problems, such as code written by multiple members of the complexity of the problem. The more errors in the system, the more errors in the software. Therefore, we need to ensure that the quality of the software is reliable, the system for all aspects of the analysis, including how to find and correct the problem through technical review.

The software testing should be carried out in strict accordance with the steps shown in Figure 2, and the general subsystem is composed of different modules, and then the software is composed of 
multiple subsystems. Each step in the test is a continuation of the previous step, and a step can also be performed at the same time. The following is the introduction of the relevant tests:

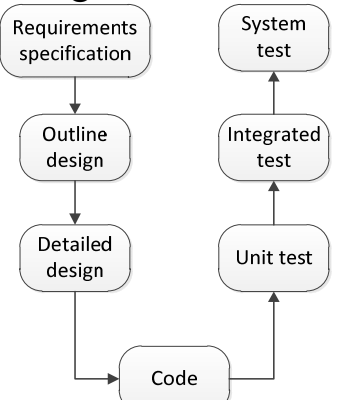

Figure 2 basic steps in software testing

The unit test, mainly to test the detailed design and program design of the problems found, in order to ensure the smooth operation of each module itself.

The integration testing, testing of each subsystem module interface to achieve coordination and smooth communication between each module. The testing of each module lays the foundation for the test of the subsystem. During the test, the test modules are put together and form a subsystem.

HAZOP system testing, mainly in accordance with the instructions to verify the function of the system is in accordance with the requirements and to test the errors appearing in the program design and system design. The specific method is to assemble and test the subsystems in the integration test, and then test the next step.

\section{Summary}

This paper is based on the JavaEE experimental teaching management system not only has a good management, portability and scalability, but also in the security, stability, handling capacity has been significantly improved. The system's development of the time is short and I have a limited level, make the system's statistical functions and produce reports and other functions have not been realized, in addition to the system of concurrent control and security mechanisms are also considered in the local, the system is not strong, but also to improve.

\section{Reference}

[1] Jie He, YishuangGeng, Fei Liu, Cheng Xu, CC-KF: Enhanced TOA Performance in Multipath and NLOS Indoor Extreme Environment, IEEE Sensor Journal, 14(11), 3766-3774, Nov. 2014

[2] $\mathrm{Na} \mathrm{Lu}$, Caiwu Lu, Zhen Yang, YishuangGeng, Modeling Framework for Mining Lifecycle Management, Journal of Networks, 9(3), 719-725, Jan. 2014

[3] YishuangGeng, KavehPahlavan, On the accuracy of rf and image processing based hybrid localization for wireless capsule endoscopy, IEEE Wireless Communications and Networking Conference (WCNC), Mar.2015

[4] Li X, Lv Z, Hu J, et al. Traffic management and forecasting system based on $3 \mathrm{~d}$ gis[J]. Cluster, Cloud and Grid Computing (CCGrid), 2015 15th IEEE/ACM International Symposium on, 2015: 991-998. 